\title{
Simulation of queue length and vehicle delays on signal-controlled intersection
}

\author{
Evgeny Leverents ${ }^{1 *}$, Roman Andronov ${ }^{1}$ and Tatiyana Anufrieva ${ }^{1}$ \\ ${ }^{1}$ Tyumen Industrial University, Volodarskogo str., 38, Tyumen, 625000, Russia
}

\begin{abstract}
The extensive use of information technology in the field of traffic control will increase the traffic capacity of intersection points and transports. To assess the efficacy of changing options for the road traffic organization or the reorganization of intersection points, you need to know the average delay in vehicles and the length of the queue. The adaptive traffic light control is one of such tools. Simulation modeling of traffic flows use for the definition of its work. The aim of this work is to create a simulation model of controlled intersection, which can evaluate the efficiency of the application the adaptive regulation in various traffic situations, including the availability or deficiency of pedestrian traffic through the intersection. The numerical experiment in in the model pass with using of the Monte Carlo method, which can to draw a conclusion about the calculated parameter on the basis of the result of the reproduction of the calculation model.
\end{abstract}

\section{Introduction}

The last years are define by the implementation of information technologies in various aspects of the existence and development of society. With regard to the urban realm, these technologies consist of a extensive use of navigation systems for transport along urban streets, the introduction of automated traffic control systems, synchronization with GPSnavigators, "Smart traffic lights" [1]. Frequently, it relies on the use of adaptive traffic light control, adapting to the changing parameters of traffic flows [2]. Despite this, the frequently of the fixed traffic light control regulation remains high, because there will always remain intersection points that are not equipped the specialized detectors. Also, when crossings work at high levels of congestion, the transport stream becomes almost stationary, which can considerable reduce the efficiency of adaptive control.

The bushcraft in large cities, it is necessary to solve the problems of the formation of congestion of traffic flows, because this leads to unproductive time and transport losses.

In this area, there are many solutions from reconstruction and construction of interchanges at different levels to the application of prohibitive measures to enter transport in the central parts of cities. The construction of interchanges is an expensive and should be applied in case of sufficient justification. The prohibitive measures are unpopular and as a result can lead to the opposite result - a general decrease in the quality of life.

In this area, many solutions are offered from the reconstruction and construction of interchanges at different levels to the application of the ban on the entry of transport to the

* Corresponding author: aroma77777@mail.ru 
central parts of the cities. The construction of interchanges is an expensive exercise and should be applied in case of sufficient justification. The prohibition of entry is unpopular and as a result can lead to the opposite result - a general decline in the quality of life.

When planning the road traffic organization at signal-controlled intersections of the street and road network, finding ways to optimize it, as well as justifying measures for reorganizing the type and configuration of intersections. It is important to determine the delays of vehicles and the economic losses conjunct with them. Especially it concerns with validation of the construction of multi-level interchange- costly affairs whose payback should be sufficiently validate.

Despite the fact that in current times there are several methods for determining vehicle delays and losses of time at a signal-controlled intersection $[3,4,5]$, the most widely accepted methods are based on queuing theory and assuming traffic flows according to the consistent patterns which called "the simplest", the Poisson flow. This is clearly understandable on the Webster's formula [3].

Its universal application is due to the comparative simplicity of the calculation. Also, its promulgation is ensured by the fact that it is adopted as a recognized [4].

The disadvantage of this method in the opinion of the authors of the article is that it is based on the theory of mass service and uses the Poisson flow of applications, which is incorrect for high level of congestion of streets and roads $[5,6]$. Also, this method improper performance when the intersection works with heavy traffic, that is, if there is a level of congestion close to 1.0. Under these conditions, the value of the average delay of the car tends to infinity. When the level of congestion close to 1.0 and the congestion is formed in the "rush hour", it's not applicable. In addition, the situation is complicated by the fact that congestion is a probabilistic phenomenon, which is difficult to evaluate by the average length of the queue and time of transport delay.

Recently, software packages and products such as PTV VISSIM, AnyLogic have been widespread acceptance, which allow to reliably simulate the traffic flow on the intersection points of the road network and evaluate delays, but these programs are expensive. Creators refer to Commercially Confidential and don't disclose information on the methodology and working methods of programs.

Based on the foregoing, in our opinion, it is required to create a methodology that would simulate the queue and calculate vehicle delays, on the basis of which it would further be possible to create methodological recommendations for optimizing traffic.

\section{Materials and methods}

In the proposed study, based on the consistent patterns of traffic flow, an imitation model of the movement of traffic flows at an isolated controlled intersection is proposed, followed by a numerical Monte-Carlo experiment and the determination of the queue length of vehicles, as well as the calculation of vehicle delays.

This method is taken on the basis of consistent patterns of the theory of transport flows on the probabilistic appearance of vehicles at different time steps and in connection with a considerable variability of results for the results of each simulation cycle. This requires a repetitive re-enactment of the traffic situation to obtain the final result with the required Indestructibility and validity [5].

\section{Experiment}


The methodology given in this study can be implemented in any programming language, including Python. It is possible to form a mathematical model of approach of vehicles to crossing and leaving them from the stop line using the Monte Carlo method with multiple pass of the traffic situation and reliable determination of the queue and midvalue of the transport delay [5].

The article [7] considered the formation of the queue in front of the stop line of the signalcontrolled intersection, which depending on the loading level, where defined 4 queuing schemes are:

- a queue of vehicles, some of which stop once at the intersection, part of the crossing passes without stopping (level of congestion less than 1.0);

- a queue of vehicles, among which there are no cars crossing the intersection without stopping, all cars make a one-time stop before crossing to the restrictive signal of the traffic light (level of congestion close to 1.0, is characteristic mainly for the adaptive mode of the traffic light object);

- a queue of vehicles, some of which stop twice for the forbidden traffic light signal (the beginning of the congestion formation, the level of congestion briefly exceeds 1.0);

- a queue consisting entirely of vehicles that stop at a traffic light prohibiting signal two or more times (the level of congestion for a relatively long time exceeds 1.0).

The last scheme of the transport queue corresponds to the systematic road congestion that occurs as a result of a prolonged excess of the vehicle density over the crossing capacity.

Based on the foregoing queuing schemes, one can propose a method for determining the transport delays in carrying out a numerical experiment using the Monte Carlo method, the main provisions of which are presented in [5].

The formulas for calculation of transport delays will change depending on the type of queue that is formed at each cycle of traffic signal regulation [6].

A preliminary study of a suitable number of vehicles for crossing and the measured value of traffic capacity showed that the similarity of the approach of cars during the traffic light cycle is characterized by a variation coefficient of $10 \%$. The measured value maximum number of cars that can throw away of intersection during the cycle (practical capacity) varies with a coefficient of $8 \%$.

As a regularity of the approach traffic flow distribution to the intersection, it is first proposed to adopt the normal distribution law of a random quantity. It is a result of high level of intensity of traffic, multilane carriageway, change of formation traffic, a large number of intersections of streets and road exits affect the ordering of the flow on the approach to the isolated intersection $[5,6]$. It is more convenient to describe the withdrawal of cars from the stop line by a beta-distribution.

\section{Results and discussion}

The results of the numerical experiment are shown in Figures 1 and 2. Also, the delay of one car was compared with the condition of a rigid traffic light regulation. For this, a methods based on the Webster's formula [9] is used.

As a source data, one direction of the two-way adjustable crossing with a capacity of 1600 avt / h was used, the cycle duration was $120 \mathrm{~s}$. The traffic situation was simulated for a time from $7 \mathrm{~h}$ to $20 \mathrm{~h}$ for the usual highway. Based on the data [5], the variation coefficient of the intersection capacity for the traffic light cycle equal to $8 \%$ was set, characterizing the unevenness of the passage of cars through the stop line and different time intervals between cars. This value of the coefficient of variation is comparatively inconsiderable, because the situation was modeled for the conditions of absence of interference in the form of passing 
pedestrians of the accompanying direction when making a turn and prohibiting the left turn while skipping the opposite direction.

In the process of numerical simulation, the number of model re-enactments was taken from 50 to 100, in accordance with the specified error value of not more than $10 \%$ with a $95 \%$ confidence. The results of computer modeling of the vehicle approach to crossing, leaving them from the stop line and calculation of delays are shown in Figure 1.

The simulation passed to the value of the intersection load level at "rush hour" equal to 1.05 ; since with a long work of crossing above this level (more than 30 minutes) there is a stable growth of the traffic queue before the crossing and leads to blanket refusal of drivers from the trip in this direction.

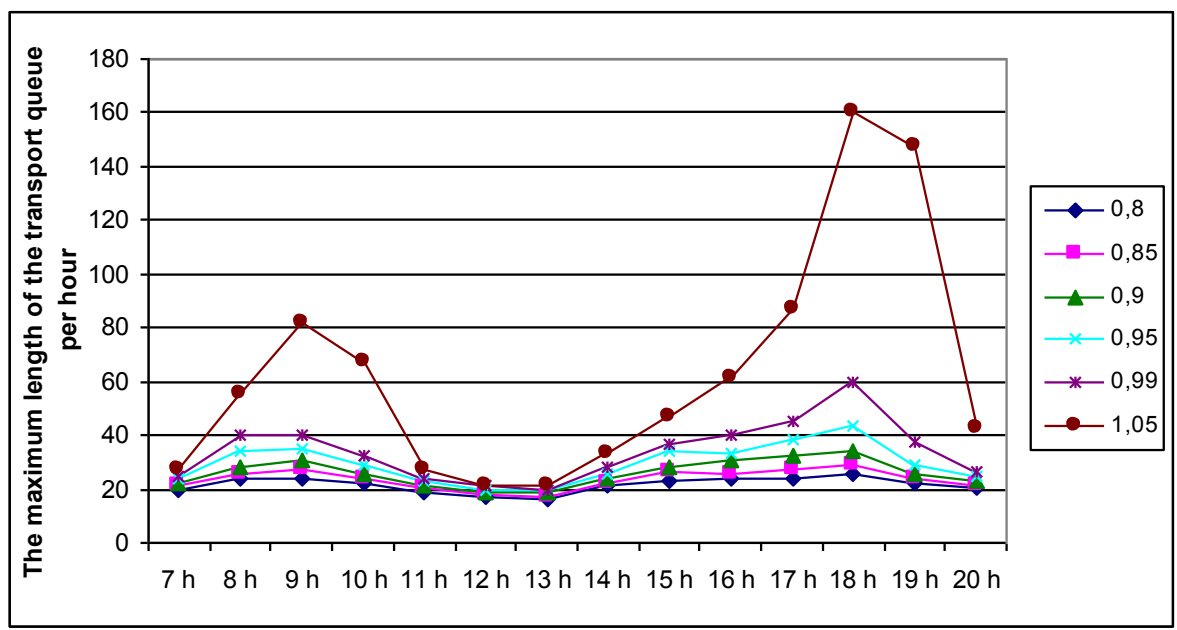

Fig. 1 -The maximum length of transport queue at different traffic congestion levels

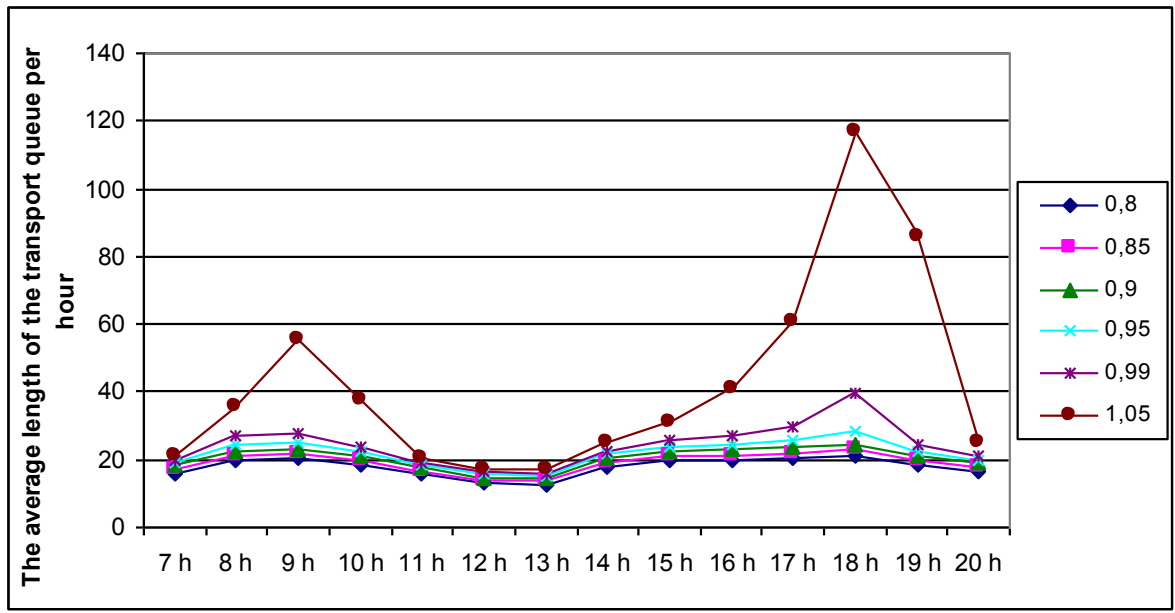

Fig. 2 -The average length of transport queue at different traffic congestion levels 


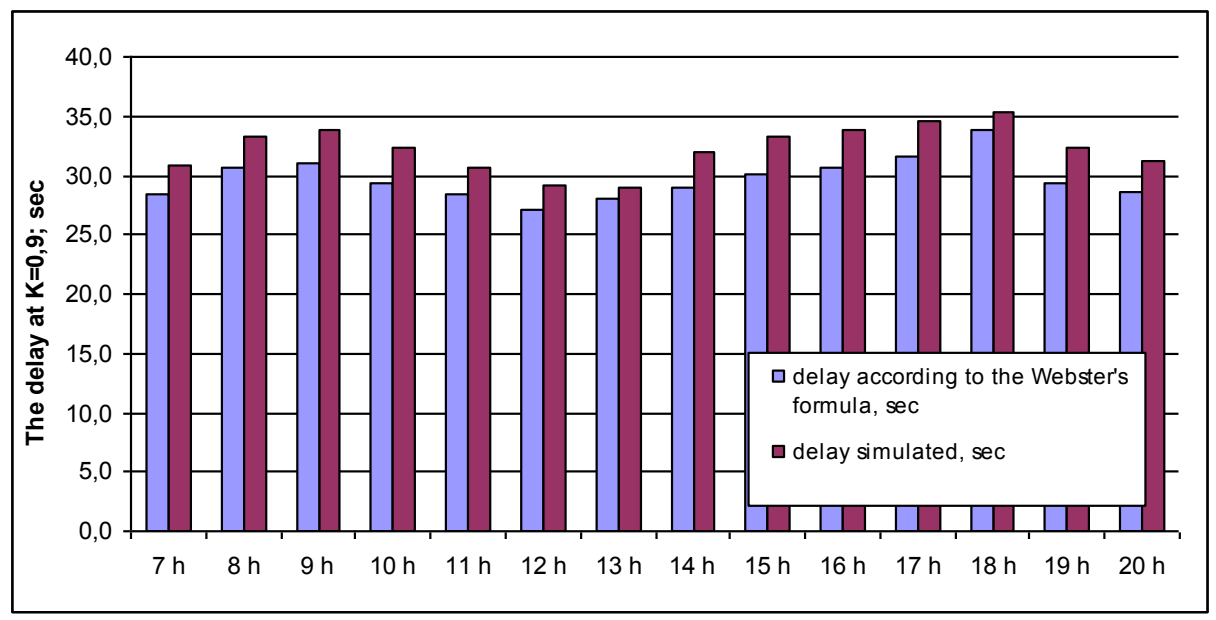

Fig. 3 The value of the average delay of the car at the traffic congestion level in the "rush hour" equal to 0.9

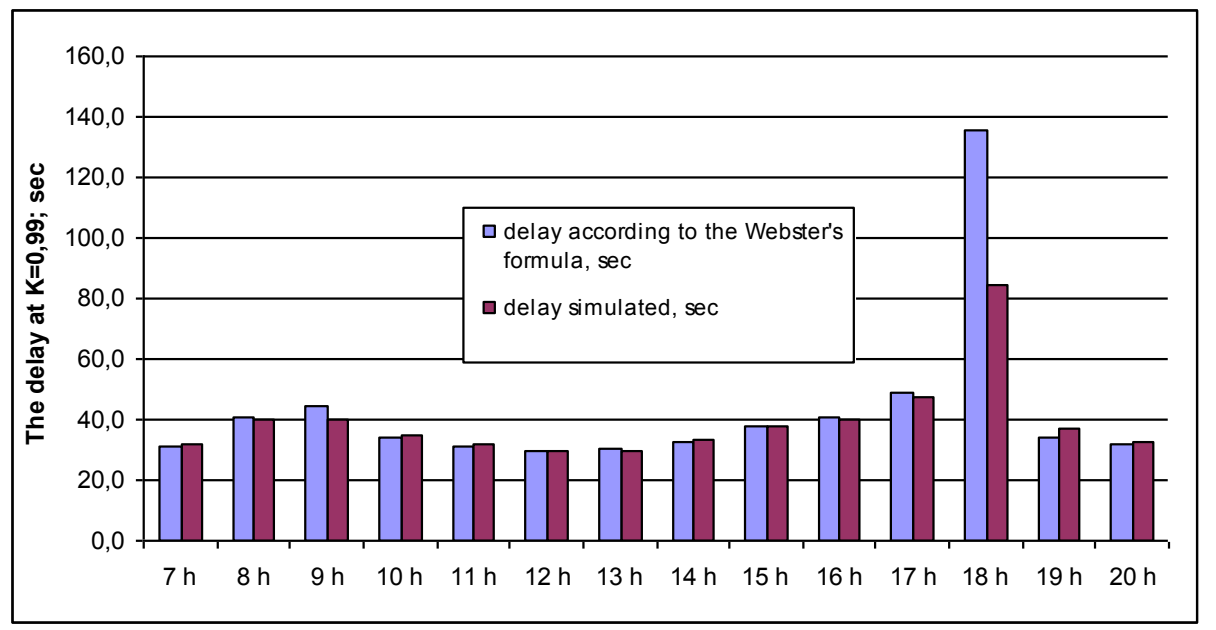

Fig. 4 The value of the average delay of the car at the traffic congestion level in the "rush hour" is 0.99 


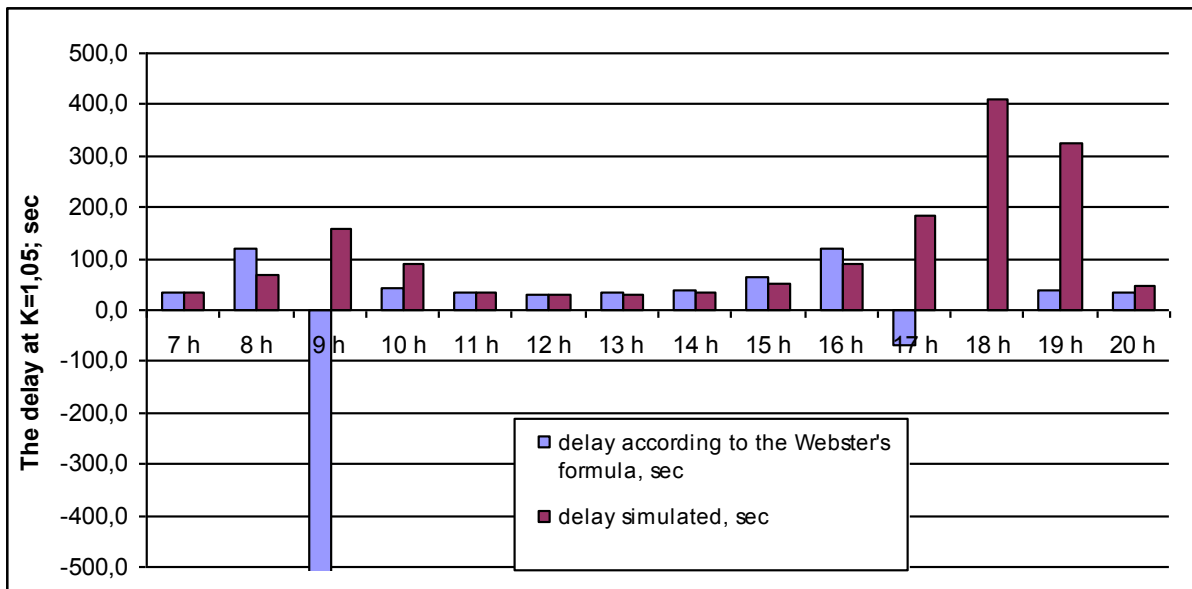

Fig. 5 The value of the average delay of the car at the traffic congestion level in the "rush hour" is 1.05

It can be seen from the diagram - when the intersection works with the traffic congestion levels of 0.95 , the values obtained as a result of the numerical experiment, values differ moderately from those calculated by the Webster's formula. Some increase in the delay can be explained by the fact that during the simulation, the deceleration and the subsequent acceleration of the car at the intersection were also taken into account as a loss of time.

Especially, a strong difference in results is evident at a traffic congestion level of about 1.0 or more, where the methods using the queuing theory and the the Poisson flow do not work. In the future, during the simulation, the parameters of the capacity were changed in the direction of increase. With the deterioration in the road traffic organization (the resolution of left-hand traffic, the transition of pedestrians in parallel with the right-turning flow, etc.), at the intersection it is assumed that the flow of traffic with a large coefficient of variation of the capacity is uneven. This will lead to an increase in the average time delay for the car (for example: the value of the queue length and the delay value with a coefficient of variation of $16 \%$ for the peak traffic congestion level of 0.99 in Figures 6 and 7).

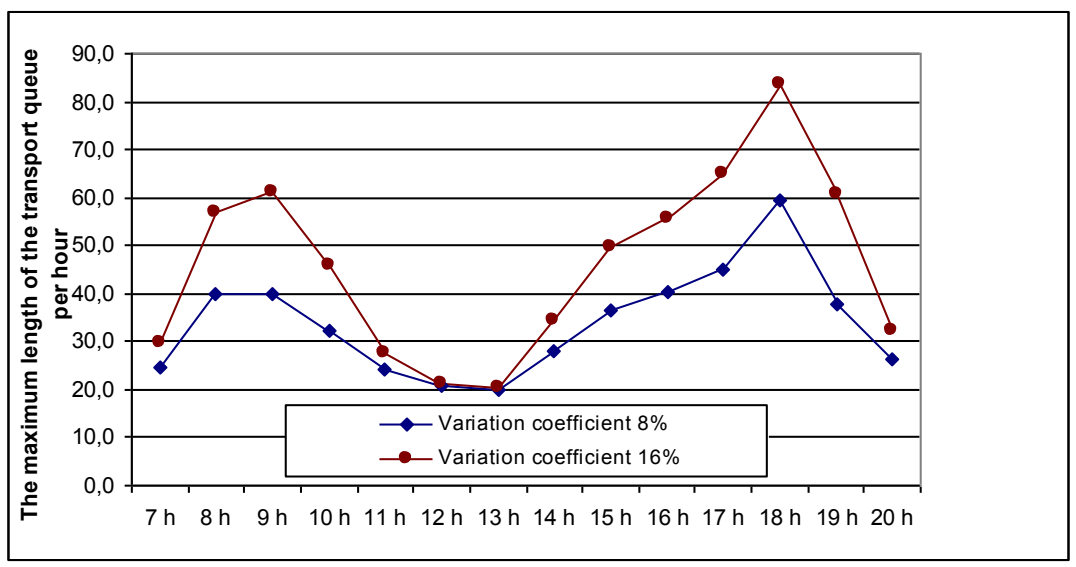

Fig. 6 -The maximum length of the vehicle queue for different values of the intersection capacity variation coefficient (traffic congestion level 0.99) 


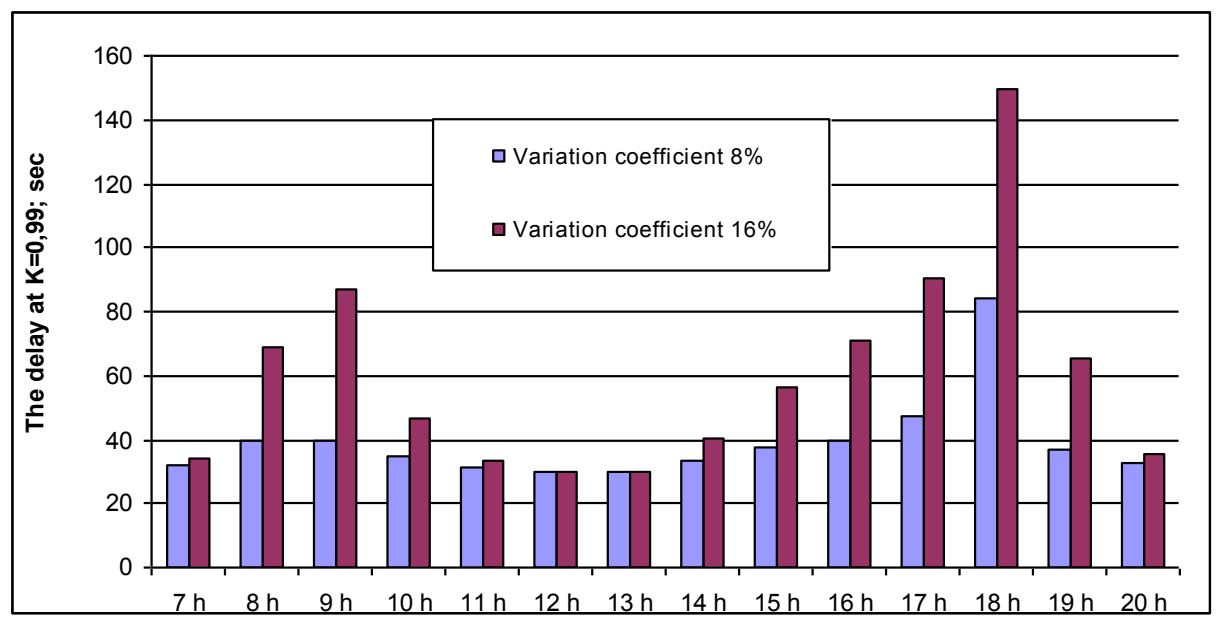

Fig. 7 The value of the average vehicle delay for different values of the variation coefficient of the crossing capacity (traffic congestion level 0.99)

\section{Conclusion}

1. For the needs of the road traffic organization and validation for the reconstruction of the signal-controlled intersection, it is necessary to reliably know the delay time of vehicles.

2. The most common and statutory methodology, based on the Webster's formula, incorrectly calculates the delay of vehicles when crossing with a load level close to 1.0. The method does not work, if necessary, to evaluate delays in the calculated "rush hour" when the traffic congestion level 1.0 is exceeded for a short time.

3. The most convenient, in our opinion, is the application of the methods described in [5] and based on the mathematical modeling of queues at a signal-controlled intersection with the help of a numerical experiment carried out by the Monte Carlo method.

4. The results of the numerical experiment showed that the value of the average delay of a stopped car at an isolated signal-controlled intersection. As it shown it increases with the greater disproportion of the approach of vehicles to the intersection and the unevenness of the actual throughput of adverse conditions of road traffic organization at the intersection (left turns in the general flow, the combination of direct and left- the movement of pedestrians in the general flow, etc.).

5. It is necessary in the future to describe in more detail the limits to applicability of the methods, to study in detail the effect of planning the signal-controlled intersection and the road traffic organization on them and the unevenness of the actual value capacity. It is also proposed to study the applicability of the methods at intersections working in coordination with nearby traffic lights or on city streets and roads, where the incoming traffic flow is influenced by nearby traffic lights.

\section{References}

1. A.K. Debnath, A methodological framework for benchmarking smart transport cities (Cities 37, 2014)

2. V. Astarita, V. P. Giofrè, V. Alessandro, A cooperative intelligent transportation system for traffic light regulation based on mobile devices as floating car data (ASRJETS) 19.1 
(2016)

3. Y. A. Kremenets, M. B. Pechersii, and M. B. Afanas' ev. "Technical means of traffic management." optional. M-tion of Education and Science Ros. Federation as a textbook. for students/YA Kremenets, MP Pechersk, MB Afanasiev.-M.: Akademkniga (2005).

4. ODM 218.6.003-2011. Metodicheskie rekomendacii po proektirovaniju svetofornyh objektov na avtomobil'nyh dorogah [Methodical recommendations on the design of traffic lights on highways] (Росавтодор, 2013)

5. R.V. Andronov, Modelirovanie ocheredej na reguliruemyh peresechenijah ulichnodorozhnoj seti krupnogo goroda $\mathrm{v}$ uslovijah plotnogo transportnogo potoka [Modeling of queues at regulated street intersections in a large city within the dense traffic] (Tyumen, 2007)

6. V. F. Babkov, Road conditions and traffic safety (1975)

7. R.V. Andronov, B.P. El'kin, Genze D.A. Concept congestion and queues forming on a regulated intersection of dense traffic flow conditions (Kazan, 2015)

8. R.V. Andronov, On methods of evaluation of traffic delays on a regulated intersection (Kazan, 2015)

9. R.V. Andronov, Calculation of economic losses of users of the street-road network at regulated intersections to justify measures for reconstruction and improvement of traffic management (Kazan, 2015). 